\title{
Measuring engagement elicited by eye contact in Human-Robot Interaction
}

\author{
Kompatsiari K., Ciardo F., De Tommaso D., Wykowska A., Member, IEEE
}

\begin{abstract}
The present study aimed at investigating how eye contact established by a humanoid robot affects engagement in human-robot interaction (HRI). To this end, we combined explicit subjective evaluations with implicit measures, i.e. reaction times and eye tracking. More specifically, we employed a gaze cueing paradigm in HRI protocol involving the iCub robot. Critically, before moving its gaze, iCub either established eye contact or not with the user. We investigated the patterns of fixations of participants' gaze on the robot's face, joint attention and the subjective ratings of engagement as a function of eye contact or no eye contact. We found that eye contact affected implicit measures of engagement, i.e. longer fixation times on the robot's face during eye contact, and joint attention elicited only after the robot established eye contact. On the contrary, explicit measures of engagement with the robot did not vary across conditions. Our results highlight the value of combining explicit with implicit measures in an HRI protocol in order to unveil underlying human cognitive mechanisms, which might be at stake during the interactions. These mechanisms could be crucial for establishing an effective and engaging HRI, and could potentially provide guidelines to the robotics community with respect to better robot design.
\end{abstract}

\section{INTRODUCTION}

\section{A. Measuring engagement in HRI}

Engagement with a robot partner is the most crucial factor in successful and natural human-robot interaction (HRI). Therefore, it is imperative to address the issue of engagement in HRI research. As stated in [1, p.1]: "Engagement is a category of user experience characterized by attributes of challenge, positive affect, endurability, aesthetic and sensory appeal, attention, feedback, variety/novelty, interactivity, and perceived user control". Studies that have examined the aspect of engagement in HRI used both explicit [e.g., 2-5] and implicit measures [6-13]. Explicit measures and questionnaires - while providing valuable hints regarding the phenomenon of interest, suffer from several limitations. First, they rely on explicit reports, meaning that participants need to be able to consciously assess their inner states. However, many mechanisms of social cognition are implicit and automatic, and thus not necessarily accessible to conscious awareness. Furthermore, explicit measures are dependent on introspective abilities and interpretation of the questions and can be prone to various biases, such as social desirability effect [14]. Finally, explicit responses are not sufficiently informative with respect to specific cognitive mechanisms involved. In natural

\footnotetext{
*The project has received funding from the European Research Council (ERC) under the European Union's Horizon 2020 research and innovation programme (grant awarded to AW, titled "InStance: Intentional Stance for Social Attunement". Grant agreement No: 715058).

All authors are with the Istituto Italiano di Tecnologia, Genova, 16145. K.K is affiliated with Ludwig-Maximilians-universität (DE).. A.W. is an
}

interactions, people are often not aware that their brains employ certain mechanisms and processes. However, thanks to the careful design of experimental paradigms inspired by research in cognitive psychology or cognitive/social neuroscience that target and focus on specific mechanisms, we can collect objective implicit metrics and draw conclusions about what specific cognitive processes are at stake [15-17]. Typically, psychologists use performance measures (reaction times, error rates) to study mechanisms of perception, cognition, and behavior, and also the social aspects thereof: for example, joint attention [e.g., 15-22], or visuo-spatial perspective taking [23-24]. As such, these measures have informed researchers about the respective cognitive processes with high reliability, and without the necessity of participants being aware of the processes under investigation. In addition to performance measures, researchers have also widely used other implicit measures - behavioral (e.g., eye tracking or motion capture) or neurophysiological/neuroimaging: for example, electroencephalogram (EEG), Galvanic skin response (GSR) or functional magnetic resonance imaging (fMRI). Those measures provide a valuable source of information regarding neural and physiological correlates of behavior.

\section{B. Joint attention as a measure of engagement in HRI}

One implicit measure of engagement with an interaction partner is joint attention (JA). JA occurs when two agents direct their focus of attention to the same object or event in the environment. This fundamental mechanism of social cognition is a basis for many other complex processes involved in social interactions. In fact, an impaired ability to engage in JA has been reported in the case of individuals diagnosed with autism spectrum disorder [25]. In humancomputer interaction (HCI) and HRI research, JA has been postulated to be a marker of engagement [6, 26]. For instance, Anzalone et al. used JA among other dynamic metrics (synchrony, imitation) to evaluate engagement in HRI [6]. Peters et al [26] defined the level of engagement between a user and virtual agent by measuring JA- i.e. how much the user has been looking at objects looked at or pointed by the virtual agent. Moreover, Kasari et al. [27] showed that JA mediated interventions increased engagement of toddlers during interaction with caregivers.

Researchers in cognitive psychology have operationalized JA in the form of the gaze cueing paradigm [18-19]. This is a

adjunct professor in Engineering Psychology, University of Technology, Luleå University of Technology. Corresponding authors: A.W., phone: +39 $0208172 \quad 242$, email: agnieszka.wykowska@iit.it, K.K., email kyveli.kompatsiari@iit.it. 
classical attentional task in which participants are typically presented with a face on the computer screen. The face initially has either eye closed or directed straight ahead. Subsequently, the direction of the gaze is shifted to one of the sides of the screen the gazed-at or a different location. Participants' task is to determine either target's identity or simply respond to its presence. When participants "engage" in JA with the "gazer" they attend to where the gazer shifts his/her eyes. Therefore, detection/discrimination of any target at the gazed-at location is faster and more accurate than at the other locations, this effect is known as the cueing effect (GCE). Recent studies showed that the GCE can be elicited in naturalistic and ecologically valid paradigms and that it is reflected, apart from performance measures, also in EEG [2830], fMRI [31-33], and eye tracking [34-35] measures.

Here, we would like to specifically focus on eye tracking as an implicit measure of engagement [6-7, 12], as eye movements are particularly informative with respect to attentional processes [36]. In the context of social interaction, eye movements not only are informative with respect to the individual's attentional focus, but they are also signaling to others where attention is oriented. As such, they are one of the most important social signals with which we convey our inner mental states.

\section{Aim of study and related work}

In this study, we aimed at examining whether eye contact established by the iCub robot [37-38] would influence engagement in HRI, measured by two implicit objective markers: JA (by means of the GCE) and patterns of fixations on the face of the robot during eye-contact. Eye contact is one of the most important social signals communicating the intention to engage in an interaction. Eye contact between humans has been shown to affect various cognitive processes such as attention or memory, and also physiological states, for example arousal [39-41].

In the context of HRI, research examining the effect of eye contact mainly focused on subjective evaluations of the robot [42-46], and how it is related to engagement [11]. In the present study, we address for the first time the impact of eye contact on two different implicit measures of engagement: the GCE and patterns of fixations on the robot face. Such measures should allow for more in-depth analysis of the cognitive mechanisms that are affected by eye contact in HRI.

Kompatsiari et al. [17] showed that eye contact established by a robot influences JA in the sense that larger GCE has been observed for eye contact condition, as compared to no eye contact condition. However, it remains to be examined and understood what specifically causes this effect. Is it because eye contact has a "freezing" effect on attentional focus, thereby causing longer disengagement times from the robot face and longer time to reallocate attentional focus to a different location? Or perhaps there are some other attention mechanisms at stake? In the current study, we address this question by employing an eye tracking methodology and investigating the patterns of fixations on the robot face in the context of eye contact and no eye contact. Answering the question of precisely what cognitive mechanisms are affected by eye contact is not only of theoretical interest, but it has also implications for robot design. If eye contact attracts attention to the face of the robot to the point that it creates delays in disengagement, it might be a positive factor for social interaction and engagement, but might impair performance in other tasks where reallocation of attentional focus is critical.

\section{METHODS}

\section{A. Participants}

In total, twenty-four healthy adults (mean age $=25.25 \pm$ 4.01, 9 female, 2 left-handed) took part in the experiment. All had normal or corrected-to-normal vision. Participants received an honorarium of 15 euros for taking part in the experiment. They were all naive with respect to the purpose of this study, and they were debriefed about the aim of the experiment at the end of the experimental session. The experiment was conducted at the Istituto Italiano di Tecnologia (Genoa, Italy). Written consent was taken from each participant before the experimental session. The study was approved by the local ethical committee (Comitato Etico Regione Liguria).

\section{B. Stimuli and Apparatus}

The experiment was performed in an isolated and noiseattenuated room. Participants were seated opposite of iCub, at the other side of a desk, while their eyes were aligned with iCub's eyes. The target stimuli were letters $\mathrm{V}$ or $\mathrm{T}\left(3^{\circ} 32^{\prime}\right.$ high, $4^{\circ} 5^{\prime}$ wide) and they were presented at two screens $(27$ inches), laterally positioned on the desk (75 cm apart, centreto-centre). The screens were tilted back (by approximately $12^{\circ}$ from the vertical position) and were rotated to the right (right screen) or left (left screen) by $76^{\circ}$. iCub's gaze was directed to five different Cartesian coordinates: restingtowards a point between the desk and participant's upper body, eye contact - towards participants' eyes, no eye contact - towards the desk, left - towards the left screen, and right towards right screen [see 17, 44 for a similar procedure].

We used the iCub's gaze controller [47] for controlling the robot's gaze, specifically the eyes and the neck. The controller uses inverse kinematics to find the eyes' and neck's poses for looking at desired Cartesian coordinates in the robot's frame. In addition, it produces joints' movements that follow a minimum-jerk velocity profile. The trajectory time for the movement of eyes and neck was set to $200 \mathrm{~ms}$ and $400 \mathrm{~ms}$ respectively. The vergence of the eyes was set to 3.5 degrees and maintained constant. The participants' eyes were detected by the robot stereo cameras using the face detector [https://github.com/robotology/human-sensing], which uses the dlib library [http://dlib.net]. The eyes detection produced valid results for $92.26 \pm 16.04 \%$ of the administered trials. When the eyes were not detected by the algorithm, the robot was programmed to look straight. Since participants were seated face-to-face with iCub and their eyes were aligned with iCub's eyes, this procedure ensured the establishment of eye contact even in the rare case of the algorithm's failure. The Cartesian coordinates of the target positions were defined according to predefined values of pitch, roll and yaw of the neck's joints. These angles were selected adequately in order to ensure balanced joints' displacements between conditions, 
i.e. a displacement of $12^{\circ}$ in the pitch between resting->eye contact and resting->no eye contact, a displacement of $27^{\circ}$ in the yaw, $12^{\circ}$ in the pitch and $7^{\circ}$ in the roll between eye contact->left or right and no eye contact-> left or right. Table 1 shows the desired and measured angles of the neck.

The measured mean trajectory times for the gaze positions were very close to the specified trajectory time for the neck movement (400 ms): Mresting-eye contact $=401.12 \pm 0.37$ (ms) and Mresting-no eye contact $=400.65 \pm 0.28(\mathrm{~ms})$. The mean trajectory times concerning the gaze shift positions were: Meye contact-shift $=404.61 \pm 2.34$ (ms) and Mno eye contact-shift $=405.54 \pm 1.43$ (ms).

\section{Procedure}

A full experimental session lasted about 40 minutes. Participants were instructed to fixate at the robot's face while performing the task. The sequence of events was the following: Each trial started with the robot having its eyes closed at the resting position. After $2 \mathrm{~s}$, the robot opened its eyes for $500 \mathrm{~ms}$. During this time, the robot extracted information related to the position of the face and the eyes of the participant without making any movement. Then, it looked either to the predefined position: down, for the condition with no eye contact, or direct to the eyes of a participant in the eye contact condition. After the movement was completed, iCub fixed its gaze to the same position for $2 \mathrm{~s}$. This means that the eye contact/no eye contact duration was 2 s. Subsequently, the robot's head and eyes shifted to either the left or the right screen. Head direction was not predictive with respect to target location (i.e. cue-target validity $=50 \%$ ). After $1000 \mathrm{~ms}$ of the onset of the robot's gaze shift, a letter appeared on one of the lateral screens. After $200 \mathrm{~ms}$, the screens turned blank until the participants' response. The trial expired if participants did not reply within $1500 \mathrm{~ms}$. The experiment consisted of 16 blocks

Table 1. Robot's gaze positions. EC represents eye contact, no EC represents no eye contact

\begin{tabular}{|lcc|c|}
\hline & \multicolumn{3}{c}{ Desired } \\
Positions & roll & pitch & yaw \\
\hline Resting & 0.0 & -12.0 & 0.0 \\
\hline EC & 0.0 & 0.0 & 0.0 \\
\hline No EC & 0.0 & -24.0 & 0.0 \\
\hline Left & -7.0 & -12.0 & 27.0 \\
\hline Right & 7.0 & -12.0 & -27.0 \\
\hline & & Measured & yaw \\
\hline Positions & roll & pitch & $-0.02 \pm 0.08$ \\
\hline Resting & $0.09 \pm 0.05$ & $-12.75 \pm 0.02$ & $-0.19 \pm 0.86$ \\
\hline EC & $0.14 \pm 0.08$ & $-0.28 \pm 0.59$ & $-0.002 \pm 0.01$ \\
\hline No EC & $-0.04 \pm 0.03$ & $-24.01 \pm 0.05$ & $27.56 \pm 0.12$ \\
\hline Left & $-7.18 \pm 0.08$ & $-12.13 \pm 0.08$ & $-27.65 \pm 0.11$ \\
\hline Right & $6.93 \pm 0.03$ & $-12.05 \pm 0.05$ & \\
\hline
\end{tabular}

of 16 trials each. A block was assigned to eye contact or no eye contact condition. The order of the blocks was counterbalanced across participants, starting either with a no eye contact block or with an eye contact block. Cue-target validity was randomized across blocks (i.e. cue-target validity $=50 \%$ in each block). At the end of each block, participants were asked to rate their engagement level with the robot on a 10-point Likert scale ( 1 = Strongly not engaged; $10=$ Strongly engaged).

\section{Eye Tracker recordings}

Eye movements were recorded using a wearable eye tracker Tobii pro glasses 2 at $100 \mathrm{~Hz}$. The head unit of Tobii pro glasses comprises of two eye cameras per eye, allowing for recording of pupil positions binocularly. The eye tracking technology is based on pupil center corneal reflection (PCCR) and dark pupil tracking. A full-HD scene camera (1920 x 1080 pixels at $25 \mathrm{fps}$ ) is embedded on the head unit with a field of view of $90^{\circ}, 16: 9$.

\section{E. Analysis}

Three participants were excluded from the analysis due to eye movement recording issues, i.e two recordings could not be opened with the Tobii pro lab software, and in one recording the iCub's face was not fully inside the field of view of the participant. One participant was excluded from the analysis, as s/he failed to follow task instructions (i.e. $\%$ of fixation on iCub's face was at the chance of level). The analysis were run on a final sample size of $\mathrm{N}=20$.

\section{1) Eye Tracker}

Firstly, we defined our Area of Interest (AOI) as iCub's face. The AOI was defined independently for data collected across the two experimental conditions since the image of iCub's face is different (eye contact: looking straight, no eye contact: looking down). Participants' raw gaze data were mapped inside or outside the desired AOI using the default mapping algorithm of Tobii Pro lab. Fixations were extracted using the default parameters of the fixation filter in Tobii Pro lab for the majority of the parameters (Tobii I-VT fixation filter [48]). Specifically, the gap fill-in interpolation was not applied the noise was removed by a moving median filter of 3 samples, the window length of the velocity calculator was set to $20 \mathrm{~ms}$, the velocity threshold was set to $30 \%$, and adjacent fixations were not merged. However, we lowered the threshold of the default value regarding the minimum fixation duration from $60 \mathrm{~ms}$ to $30 \mathrm{~ms}$ in order to extract also very short fixations.

For each trial, we extracted the number of fixations within the AOI and their duration in ms for the gaze condition phase (i.e. the time between resting and lateral movement $2000 \mathrm{~ms}$ ). If the trial belonged to the eye contact condition, the data were mapped to the AOI of iCub looking straight. In the same way, if the trial belonged to the no eye contact condition, the data were mapped to the AOI of iCub looking down. Paired sample t-tests were performed to test the statistical difference 
between eye contact and no eye contact conditions regarding the percentage of fixations and the fixations' duration inside our AOI, i.e. iCub's face.

\section{2) Behavioral Data}

The errors were $3.2 \% \pm 2.1 \%$ of the administered trials, and they were not further analyzed. RTs faster than $100 \mathrm{~ms}$ or 2.5 SDs above- or below an individual's mean for each experimental condition were removed $(2.34 \%$ of the correct trials). After removing all outliers, the experimental conditions (eye contact-valid, eye contact-invalid, no eye contact-valid, no eye contact-invalid) consisted of a similar number of trials on average, equal to $60.5 \pm 1.94$. Paired sample t-tests were conducted separately for the eye contact and no eye contact conditions between valid and invalid trials.

\section{3) Self-report ratings}

Mean engagement ratings for eye contact and no eye contact blocks were analyzed using a Wilcoxon signed-rank test.

\section{RESULTS}

\section{1) Eye Tracker}

Paired sample t-tests showed significant differences in the fixation durations between the eye contact and no eye contact condition, $\mathrm{t}(19)=-2.3, \mathrm{p}=.03,95 \%$ CI $[-390.51,-18.73]$. Specifically, fixation durations were longer for the eye contact $(M=1450.31 \mathrm{~ms}, \mathrm{SEM}=225.93 \mathrm{~ms})$ compared to the no eye contact condition $(M=1245.69 \mathrm{~ms}, \mathrm{SEM}=158.7 \mathrm{~ms})$, see Figure 1, lower panel. No difference between eye contact
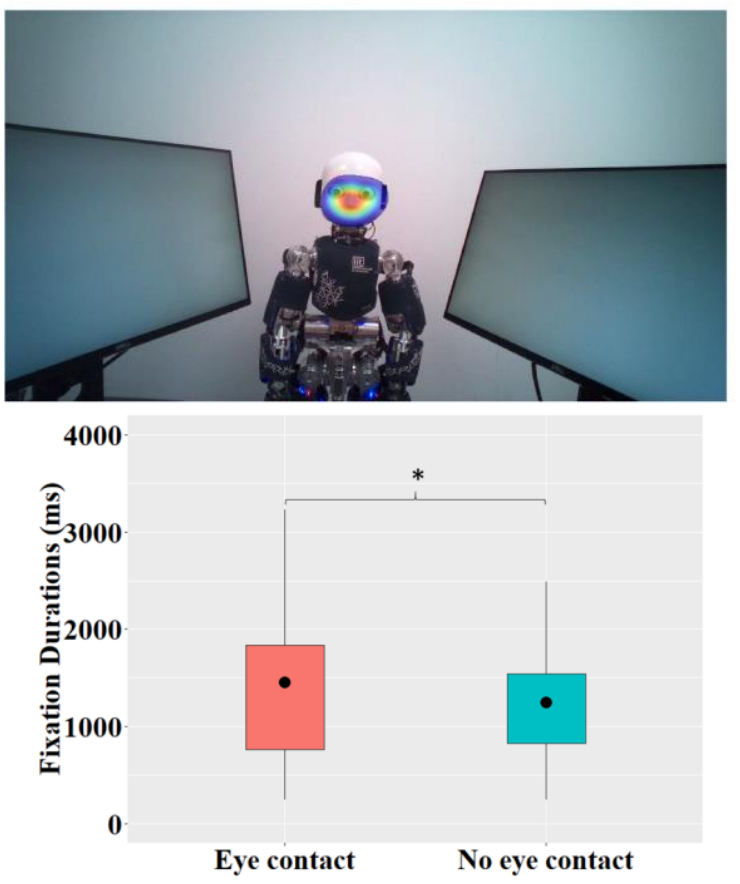

Figure 1. Upper panel: Heat map based on fixation duration values of all participants in the eye contact condition performed with PyGaze [49]. 'Red zone' represents areas to which participants performed the longest fixations. 'Blue zone' represents areas to which participants performed the shortest fixations. Lower panel: Mean fixation durations across gaze conditions. The circle represents the mean of the data. End of the whiskers represent the lowest and maximum data point within 1.5 interquartile range of the lower and upper quartile respectively. Asterisk represents significant differences between conditions.

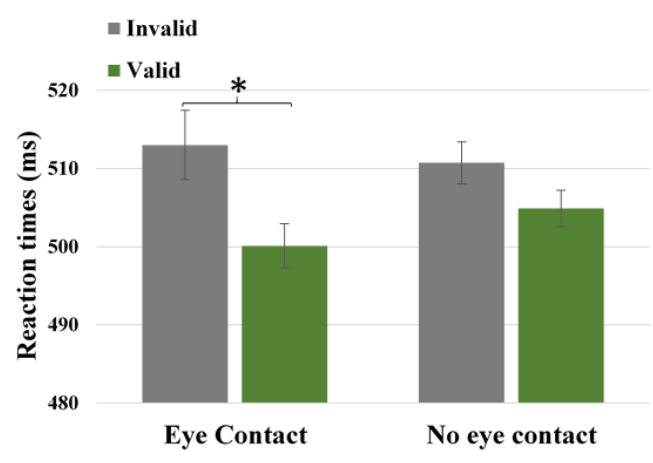

Figure 2. Means RTs across gaze conditions. Grey bars: invalid trials, green bars: valid trials. Error bars represent standard error of the means adjusted to within-participant designs according to Cousineau [50]. Asterisk represents significant differences between conditions.

and no eye contact conditions was found for the percentage of fixations inside the AOI; $\mathrm{t}(19)<1$ (eye contact: $\mathrm{M}=95.03 \%$, $\mathrm{SEM}=1.1 \%$; no eye contact: $\mathrm{M}=95.3 \%, \mathrm{SEM}=1.3 \%)$.

\section{2) Behavioral data}

Pairwise comparisons showed a significant difference between valid and invalid trials (the classical GCE) for the eye contact condition, $\mathrm{t}(19)=2.37, \mathrm{p}=.03,95 \%$ CI 1.58, 24.19], with RTs faster for valid $(\mathrm{M}=500.11 \mathrm{~ms}, \mathrm{SEM}=12.74 \mathrm{~ms})$ than invalid trials $(M=512.99 \mathrm{~ms}, \mathrm{SEM}=15.62 \mathrm{~ms})$, see Figure 2. No differences in RTs between valid and invalid trials were found for the no eye contact condition; $\mathrm{t}(19)=1.6$, $\mathrm{p}=.1195 \%$ CI $[-1.61,13.3]$ (Valid: $\mathrm{M}=504.9 \mathrm{~ms}, \mathrm{SEM}=$ $14.75 \mathrm{~ms}$; Invalid: $\mathrm{M}=510.73 \mathrm{~ms}, \mathrm{SEM}=15.2 \mathrm{~ms}$ ).

\section{3) Self-report ratings}

Participants' mean engagement ratings did not differ between the eye contact and the no eye contact condition, $\mathrm{Z}=$ $-1.72, \mathrm{p}=.09$ (eye contact: $\mathrm{M}=6.52, \mathrm{SD}=1.96$; no eye contact: $\mathrm{M}=6.17, \mathrm{SD}=1.98)$, see Figure 3 .

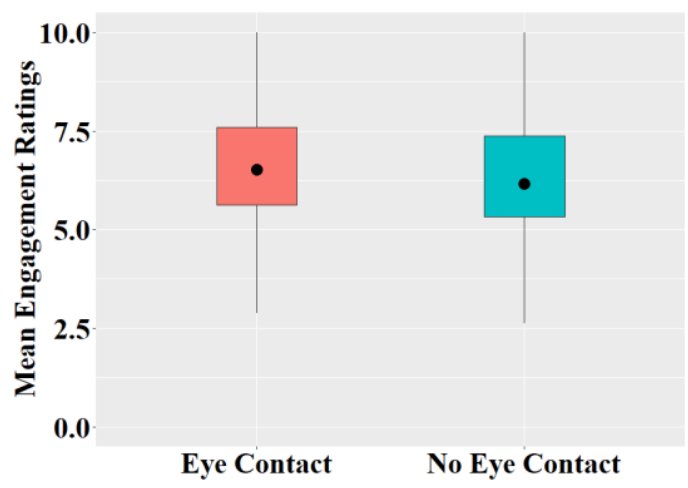

Figure 3. Mean engagement ratings across gaze conditions. The circle represents the mean of the data. End of the whiskers represent the lowest and maximum data point within 1.5 interquartile range of the lower and upper quartile respectively.

\section{DISCUSSION}

In the present study, we examined what cognitive mechanisms are at stake during eye contact established by the 
iCub humanoid robot in HRI. To this end, we combined explicit (subjective reports) and implicit measures of engagement (GCE and fixations' patterns on iCub's face).

Results showed that objective measures of engagement differed between the eye contact and the no eye contact condition. First, our results showed that participants fixated longer to iCub's face during eye contact compared to no eye contact condition. Second, we found a statistically significant GCE (i.e., faster responses to validly- compared to invalidlycued targets) only when the robot established eye contact before shifting the gaze. Such a result indicates that participants engaged in JA with iCub only when the robot established eye contact with them. It should be noted that the magnitude of the GCE in eye contact condition is comparable to what has been reported in screen-based paradigms in experimental psychology [18-22].

Results from objective measures extend recent findings related to the influence of eye contact on JA [17], and they give more insights on the cognitive mechanisms associated with this mechanism in HRI [43]. Specifically, the longer fixations duration reported in the eye contact condition suggests that when the robot established eye contact participants looked longer at its face. This might have increased the amount of attentional resources allocated at robot's face resulting in a difficulty to "disengage" from the task-irrelevant information, i.e. the head/eyes. Thus, as consequence, when the robot shifted the head/gaze laterally, participants could not disengage from its face and oriented their attention in the same direction. This resulted in faster reaction times when the target appeared at the gazed-at location compared to when it occurred in the opposite location. On the other hand, when no eye contact was established, participants looked shorter to the robot's face. Shorter fixations at iCub's face may have facilitated participants to allocate their attentional focus to the relevant target letter.

The impact of eye contact on social interaction by holding attention to the robot's face is presumably a facilitating factor in engagement and social interaction with the robot. Indeed, knowing that eye contact keeps or "freezes" attentional focus on the robot face is crucial when designing behaviors in which the robot has to grab users' attention. For instance, imagine a robot designed to give directions to the users, it should be designed to establish eye contact with the users in order to attract their attention. However, in other tasks, for example, when moving a heavy object together with the robot, focusing attention on the robot's face/eyes robot could impair the user's performance by delaying shift of attention toward, for example, a potential obstacle.

Interestingly, explicit measures of engagement were not affected by eye contact in this study, which is in contrast to findings of Experiment 1 in [17], where results from subjective and objective measures were aligned. A disassociation between explicit and implicit measures found here has been also found in Experiment 2 of [17]. In that experiment, the results showed that when the head/gaze direction of the robot was counter-predictive with respect to the target location (25\% validity) GCE and subjective ratings of engagement showed an opposite pattern. Specifically, while no GCE occurred in the eye contact condition (given to the counter-predictive nature of the head/gaze cue), participants rated their engagement lower than in the no eye contact condition. A dissociation between objective and subjective measures was also reported in [51]. These findings suggest - as argued earlier - that subjective measures are sometimes not sensitive enough to capture various (often implicit) cognitive processes involved in a task, and that effective evaluation of engagement in HRI needs to supplement subjective reports with objective measures.

In conclusion, our study highlights the necessity of using objective measures to target implicit social cognitive mechanisms that are evoked during HRI. This approach is essential for designing robot behaviors which would need to elicit or inhibit these mechanisms dependent on the specific context of the human-robot interaction.

\section{REFERENCES}

[1] H.L. O'Brien, E.G. Toms, "What is user engagement? A conceptual framework for defining user engagement with technology", Journal of the American society for Information Science and Technology, 2008, 59(6):938-55.S.M.

[2] A. Ben-Youssef, C. Clavel, S. Essid, M. Bilac, M. Chamoux, A. Lim, "UE-HRI: a new dataset for the study of user engagement in spontaneous human-robot interactions". In Proceedings of the 19th ACM International Conference on Multimodal Interaction, 2017, pp. 464-472, ACM.

[3] S. Ivaldi, S. Lefort, J. Peters, M. Chetouani, J. Provasi, E. Zibetti, "Towards engagement models that consider individual factors in HRI: On the relation of extroversion and negative attitude towards robots to gaze and speech during a human-robot assembly task". Int. J. Soc. Rob., 2017, 9(1):63-86.

[4] T. Nomura, T. Kanda, T. Suzuki, K. Kato, "Prediction of human behavior in human--robot interaction using psychological scales for anxiety and negative attitudes toward robots". IEEE transactions on robotics, 2008, 24(2):442-51.

[5] V. Rousseau, F. Ferland, D. Létourneau, F. Michaud, "Sorry to interrupt, but may I have your attention?: preliminary design and evaluation of autonomous engagement in HRI". J Hum Robot Interact, 2013, 2(3):41-61.

[6] Anzalone, S. Boucenna, S. Ivaldi, M. Chetouani, "Evaluating the engagement with social robots", Int. J.Soc. Rob., 2015, 7(4):465-78.

[7] P. Baxter, J. Kennedy, A.L. Vollmer, J. de Greeff, T. Belpaeme, "Tracking gaze over time in HRI as a proxy for engagement and attribution of social agency". In Proceedings of the 2014 ACM/IEEE international conference on Human-robot interaction, 2014, pp. 126127, ACM.

[8] J. Hall, T. Tritton, A. Rowe, A. Pipe, C. Melhuish, U. Leonards, "Perception of own and robot engagement in human-robot interactions and their dependence on robotics knowledge", Rob Autonom Syst., 2014, 62(3):392-9.

[9] S. Ivaldi, S. Lefort, J. Peters, M. Chetouani, J. Provasi, E. Zibetti, "Towards engagement models that consider individual factors in HRI: On the relation of extroversion and negative attitude towards robots to gaze and speech during a human-robot assembly task", Int J Soc Rob., 2017, 9(1):63-86.

[10] C. Lee, N. Lesh, C.L. Sidner, L.P. Morency, A. Kapoor, T. Darrell, E. Mower, D.J. Feil-seifer, "Investigating implicit cues for user state estimation in human robot interaction". In Proceedings of the International Conference on Human-Robot Interaction, 2007.

[11] C. Rich, B. Ponsler, A. Holroyd, C.L. Sidner, "Recognizing engagement in human-robot interaction", In 2010 5th ACM/IEEE International Conference on Human-Robot Interaction (HRI), 2010, pp. 375-382. IEEE.

[12] C.L. Sidner, C.D. Kidd, C. Lee, N. Lesh, "Where to look: a study of human-robot engagement", In Proceedings of the 9th international conference on Intelligent user interfaces, 2004, pp. 78-84. ACM. 
[13] M. Székely, J. Michael, "Investing in commitment: Persistence in a joint action is enhanced by the perception of a partner's effort", Cogn. 2018, 1;174:37-42.

[14] D.G. Humm, K.A. Humm, "Validity of the Humm-Wadsworth Temperament Scale: with consideration of the effects of subjects' response-bias, J Psychol., 1944, 1;18(1):55-64.

[15] A. Wykowska, J. Kajopoulos, M. Obando-Leitón, S.S. Chauhan, J.J. Cabibihan, G. Cheng, "Humans are well tuned to detecting agents among non-agents: examining the sensitivity of human perception to behavioral characteristics of intentional systems", Int. J. Soc. Rob., 2015, 1;7(5):767-81.C.K.

[16] A. Wykowska, E. Wiese, A. Prosser, H.J. Müller., "Beliefs about the minds of others influence how we process sensory information", PLoS One, 2014, 8;9(4):e94339.

[17] K. Kompatsiari, F. Ciardo, V. Tikhanoff, G. Metta, A. Wykowska, "On the role of eye contact in gaze cueing", Sci Rep., 2018, 8(1):17842.

[18] Friesen, A. Kingstone, "The eyes have it! Reflexive orienting is triggered by nonpredictive gaze", Psychon. Bull. Rev., 1998, 1;5(3):490-5.

[19] I.V. J. Driver, G. Davis, P. Ricciardelli, P. Kidd, E. Maxwell, S. BaronCohen, "Gaze perception triggers reflexive visuospatial orienting", Vis cogn., 1999, 6(5):509-40.

[20] Perez-Osorio, J., Müller, H. J., Wiese, E. \& Wykowska, A. "Gaze following is modulated by expectations regarding others' action goals" PLoS One 10, 2015.

[21] E. Wiese, A. Wykowska, J. Zwickel, H.J. Müller, "I see what you mean: how attentional selection is shaped by ascribing intentions to others", PloS one, 2012, 7(9):e45391.

[22] F. Ciardo, P. Ricciardelli, L. Lugli, S. Rubichi, C. Iani. "Eyes keep watch over you! Competition enhances joint attention in females", Acta Psychol., 2015, 1;160:170-7.

[23] D. Samson, I.A. Apperly, J.J. Braithwaite, B.J. Andrews, S.E. Bodley Scott "Seeing it their way: evidence for rapid and involuntary computation of what other people see", $J$ Exp Psychol Hum Percept Perform., 2010, 36(5):1255.

[24] J. Zwickel, S.J. White, D. Coniston, A. Senju, U. Frith, "Exploring the building blocks of social cognition: spontaneous agency perception and visual perspective taking in autism", Soc Cogn Affect Neurosci, 2010, 6(5):564-71.

[25] S. Baron-Cohen, "Mindblindness: An essay on autism and theory of mind", MIT press, 1997.

[26] C. Peters, S. Asteriadis, K. Karpouzis, "Investigating shared attention with a virtual agent using a gaze-based interface", J Multimodal User In, 2010, 3(1-2):119-30.

[27] C. Kasari, A.C. Gulsrud, C. Wong, S. Kwon, J. Locke, "Randomized controlled caregiver mediated joint engagement intervention for toddlers with autism". J Autism Dev Disord., 2010, 40(9):1045-56.

[28] J. Perez-Osorio, H.J. Müller, A. Wykowska, "Expectations regarding action sequences modulate electrophysiological correlates of the gazecueing effect", Psychophysiol., 2017, 54(7):942-54.

[29] A.M. Schuller, B. Rossion., "Spatial attention triggered by eye gaze increases and speeds up early visual activity", Neuroreport, 2001, 8;12(11):2381-6.

[30] K. Kompatsiari, J. Pérez-Osorio, D. De Tommaso, G. Metta, A. Wykowska, "Neuroscientifically-grounded research for improved human-robot interaction", In 2018 IEEE/RSJ International Conference on Intelligent Robots and Systems, IROS, 2018, pp. 34033408). IEEE.

[31] C. Özdem, E. Wiese, A. Wykowska, H. Müller, M. Brass, F. Van Overwalle, "Believing androids-fMRI activation in the right temporoparietal junction is modulated by ascribing intentions to non-human agents", Soc Neurosci, 2017, 12(5):582-93.

[32] A. Kingstone, C. Tipper, J. Ristic, E. Ngan, "The eyes have it!: An fMRI investigation”, Brain Cogn., 2004, 1;55(2):269-71.

[33] J.K. Hietanen, L. Nummenmaa, M.J. Nyman, R. Parkkola, H. Häm"äläinen, "Automatic attention orienting by social and symbolic cues activates different neural networks: An fMRI study, Neuroimage. 2006, 15;33(1):406-13.

[34] F. Ciardo, B.F. Marino, R. Actis-Grosso, A. Rossetti, P. Ricciardelli, "Face age modulates gaze following in young adults", Sci Rep., 2014, 4:4746.
[35] U.J. Pfeiffer, K. Vogeley, L. Schilbach, "From gaze cueing to dual eyetracking: novel approaches to investigate the neural correlates of gaze in social interaction", Neurosci Biobehav Rev., 2013, 37(10):2516-28.

[36] H. Deubel, W.X. Schneider, "Saccade target selection and object recognition: Evidence for a common attentional mechanism", Vision Res., 1996, 36(12):1827-37.

[37] G. Metta, L. Natale, F. Nori, G. Sandini, D. Vernon, L. Fadiga, C. von Hofsten, K. Rosander, M. Lopes, J. Santos-Victor, A. Bernardino, L. Montesano L, "The iCub humanoid robot: An open-systems platform for research in cognitive development". Neural Networks, 2010, 23(89): $1125-1134$.

[38] L. Natale, C. Bartolozzi, D. Pucci, A. Wykowska, G. Metta, "The notyet-finished story of building a robot child", Science Robotics, 2017, 2 (13).

[39] A. Senju, T. Hasegawa, "Direct gaze captures visuospatial attention", Vis Cog., 2005, 12(1):127-44.

[40] A.F. Hamilton, "Gazing at me: the importance of social meaning in understanding direct-gaze cues", Philos. Trans. Royal Soc. B: Biological Sciences, 2016, 371(1686):20150080.

[41] M. Dalmaso, L. Castelli, G. Galfano, "Attention holding elicited by direct-gaze faces is reflected in saccadic peak velocity, Exp Brain Res., 2017, 235(11):3319-32.

[42] M. Imai, T. Kanda, T. Ono, H. Ishiguro, K. Mase "Robot mediated round table: Analysis of the effect of robot's gaze", In Proceedings. 11th IEEE International Workshop on Robot and Human Interactive Communication, 2002, pp. 411-416, IEEE.

[43] H. Admoni, B. Scassellati, "Social eye gaze in human-robot interaction: A review". J Hum Robot Interact, 2017, 6(1):25-63.

[44] K. Kompatsiari, V. Tikhanoff, F. Ciardo, G. Metta, A. Wykowska. "The importance of mutual gaze in human-robot interaction", In International Conference on Social Robotics, 2017, pp. 443-452, Springer, Cham.

[45] T. Yonezawa, H. Yamazoe, A. Utsumi, S. Abe, "Gaze-communicative behavior of stuffed-toy robot with joint attention and eye contact based on ambient gaze-tracking", In Proceedings of the 9th international conference on Multimodal interfaces, 2007, pp. 140-145. ACM

[46] Y. Zhang, J. Beskow, H. Kjellström, "Look but Don't Stare: Mutual Gaze Interaction in Social Robots", In International Conference on Social Robotics, 2017, pp. 556-566. Springer, Cham.

[47] A. Roncone, U. Pattacini, G. Metta, and L. Natale, "A Cartesian 6-DoF Gaze Controller for Humanoid Robots.," in Proceedings of Robotics: Science and Systems, 2016.

[48] A. Olsen, "The Tobii I-VT Fixation Filter - Algorithm description", Retrieved from http://www.tobii.com/eye-trackingresearch/global/library/white-papers/the-tobii-i-vt-fixation-filter/, 2012.

[49] Dalmaijer, E.S., Mathôt, S., \& Van der Stigchel, S. (2013). PyGaze: an open-source, cross-platform toolbox for minimal-effort programming of eye tracking experiments. Behav Res Meth, doi:10.3758/s13428-013$0422-2$

[50] D. Cousineau, "Confidence intervals in within-subject designs: A simpler solution to Loftus and Masson's method," Tutorials in quantitative methods for psychology, 1(1), 42-45, 2005.

[51] M.C. Martini, G.A .Buzzell \& E. Wiese, "Agent Appearance Modulates Mind Attribution and Social Attention in Human-Robot Interaction", in International Conference in Social Robotics, 2015 431-439. 